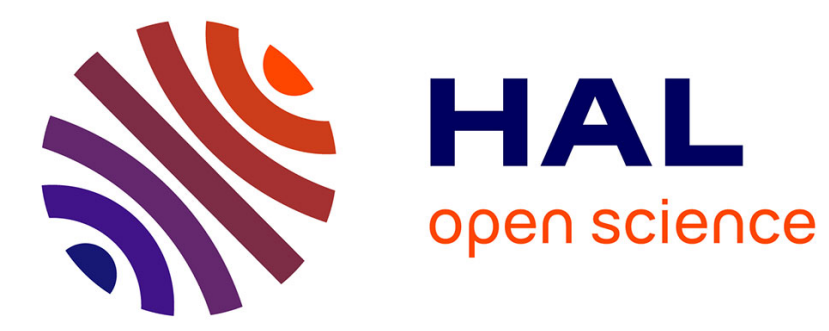

\title{
Voltage transient analysis as a generic tool for solar junction characterization
}

Ari Bimo Prakoso, Chenjin Lu, C Rusli, Daniele Cortecchia, Cesare Soci, Maxime Berthe, D. Deresmes, Boubakeur Ayachi, Jean-Pierre Vilcot, Heinrich

Diesinger

\section{To cite this version:}

Ari Bimo Prakoso, Chenjin Lu, C Rusli, Daniele Cortecchia, Cesare Soci, et al.. Voltage transient analysis as a generic tool for solar junction characterization. Journal of Physics D: Applied Physics, 2018, 51 (34), 10.1088/1361-6463/aad274 . hal-02331487

\section{HAL Id: hal-02331487 \\ https://hal.science/hal-02331487}

Submitted on 24 Oct 2019

HAL is a multi-disciplinary open access archive for the deposit and dissemination of scientific research documents, whether they are published or not. The documents may come from teaching and research institutions in France or abroad, or from public or private research centers.
L'archive ouverte pluridisciplinaire HAL, est destinée au dépôt et à la diffusion de documents scientifiques de niveau recherche, publiés ou non, émanant des établissements d'enseignement et de recherche français ou étrangers, des laboratoires publics ou privés. 
ACCEPTED MANUSCRIPT

\section{Voltage transient analysis as a generic tool for solar junction characterization}

To cite this article before publication: Ari Bimo Prakoso et al 2018 J. Phys. D: Appl. Phys. in press https://doi.org/10.1088/1361-6463/aad274

\section{Manuscript version: Accepted Manuscript}

Accepted Manuscript is "the version of the article accepted for publication including all changes made as a result of the peer review process, and which may also include the addition to the article by IOP Publishing of a header, an article ID, a cover sheet and/or an 'Accepted Manuscript' watermark, but excluding any other editing, typesetting or other changes made by IOP Publishing and/or its licensors"

This Accepted Manuscript is @ 2018 IOP Publishing Ltd.

During the embargo period (the 12 month period from the publication of the Version of Record of this article), the Accepted Manuscript is fully protected by copyright and cannot be reused or reposted elsewhere.

As the Version of Record of this article is going to be / has been published on a subscription basis, this Accepted Manuscript is available for reuse under a CC BY-NC-ND 3.0 licence after the 12 month embargo period.

After the embargo period, everyone is permitted to use copy and redistribute this article for non-commercial purposes only, provided that they adhere to all the terms of the licence https://creativecommons.org/licences/by-nc-nd/3.0

Although reasonable endeavours have been taken to obtain all necessary permissions from third parties to include their copyrighted content within this article, their full citation and copyright line may not be present in this Accepted Manuscript version. Before using any content from this article, please refer to the Version of Record on IOPscience once published for full citation and copyright details, as permissions will likely be required. All third party content is fully copyright protected, unless specifically stated otherwise in the figure caption in the Version of Record.

View the article online for updates and enhancements. 


\title{
Voltage transient analysis as a generic tool for solar junction characterization
}

\author{
A.-B. Prakoso ${ }^{1}$, Chenjin Lu ${ }^{1}$, Rusli $^{1}$, D. Cortecchia ${ }^{2}$, C. Soci $^{2}$, \\ M. Berthe ${ }^{3}$, D. Deresmes ${ }^{3}$, B. Ayachi ${ }^{3}$, J.-P. Vilcot ${ }^{3}$, H. \\ Diesinger $^{3}$ \\ ${ }^{1}$ Nanyang Technol. Univ., School of Electrical and Electronic Engineering, \\ Nanoelect Ctr Excellence, Novitas, 50 Nanyang Ave, Singapore 639798, Singapore \\ 2 Nanyang Technol University, School of Physical and Mathematical Sciences, \\ Division of Physics and Appl Physics, 21 Nanyang Link, Singapore 637371, Singapore \\ ${ }^{3}$ Institut d'Electronique, Microelectronique et Nanotechnologie, CNRS UMR 8520, \\ CS60069, F-59652 Villeneuve d'Ascq Cedex, France \\ E-mail: heinrich.diesinger@univ-lille.fr \\ March 2018
}

Abstract. Surface photovoltage transients on' solar junctions have often been associated with carrier lifetime in the literature. However, the carrier decay in a junction is not governed by a first order carrier decay, but resulting from a differential capacitance interacting with a differential conductivity. This phenomenon is well known as the Kane-Swanson formalism in an engineering context where the carrier density transient is measured by photoconductance with a microwave or infrared beam. In this work, we solve the same differential equations numerically to model the carrier decay in the large signal domain extending over 5 orders of carrier density. Since the surface-voltage is linked to the carrier density by a logarithmic relation, we express the carrier decay as surface photovoltage transients. We show how from photovoltage transients, the same information as from photoconductance can be drawn. To demonstrate the method as a generic tool, it is applied to 4 types of solar cells, two monocrystalline silicon cells, a Perovskite solar cell, a transition metal oxide / silicon hybrid junction, and a CIGS solar cell. Acquiring photovoltage transients by Kelvin force microscopy allows working on partial junctions without top contact, speeding up research of future photovoltaic materials. Furthermore, parameters may be mapped with a better lateral resolution compared to the millimeter resolution of microwave photoconductance.

Submitted to: J. Phys. D: Appl. Phys.

1. Carrier dynamics in solar junctions, transient photovoltage and microwave photoconductance

Carrier dynamics and lifetime measurements on solar junctions are becoming a popular charactérization technique. In nanostructured devices, the scale at which the junction 
characteristics vary reduces correspondingly, requiring new microscopic characterization tools or at least microscopic equivalents of existing techniques. Transient photovoltage decay (TPV or TPD) or open circuit voltage decay (OCVD) measurements are one approach of adressing carrier density dynamics. They have been applied macroscopically to Perovskite solar cells $[1,2]$ and to dye-sensitized solar cells (DSC) with a contactless technique using a high-impedance OpAmp voltage follower [3]. Microscopic implementations using KFM as the electrode have been used to determine carrier lifetime in polycristalline solar cells [4], in silicon nanocrystals [5], and organic photovoltaics $[6,7]$. These implementations use time-averaging methods to reconstruct a decay curve indirectly by measuring average photovoltage as function of optical intensity modulation frequency.

Another common technique in photovoltaics research and industry is the determination of the emitter saturation current of a solar junction from microwave photoconductance lifetime measurements by tracing the effective decay time over the carrier density [8]. The so-called quasi steady state (QSS) condition means that an effective carrier decay time is acquired for small signal at which the carrier density variation is small compared to the carrier density. The effective decay time derived by the Kane-Swanson (KS) formalism [9] is given by [10]

$$
\frac{1}{\tau_{\text {eff }}}-\frac{1}{\tau_{\mathrm{b}}}=\left(J_{\mathrm{oe}, \text { front }}+J_{\text {oe,back }}\right) \frac{N_{\mathrm{dop}}+\Delta n_{\mathrm{avg}}}{q W n_{\mathrm{i}, \mathrm{eff}}^{2}}
$$

Basically, the carrier decay is due to diffusion current under forward bias in response to minority carrier generation. The term $\tau_{\mathrm{b}}$ combines Auger, radiative and $\mathrm{SRH}$ recombination and the injection rate has to be chosen such that those terms remain negligible compared to the diffusion current. Then, plotting $1 / \tau_{\text {eff }}$ over $\Delta n_{\mathrm{av}}$ and determining the slope yields the sum of the emitter saturation currents $J_{\text {oe }}$, which is linked to the potential open-circiut voltage $V_{\text {oc }}$ by the relation

$$
\frac{J_{\mathrm{sc}}}{J_{\mathrm{oe}}}=\exp \left(\frac{e V_{\mathrm{oc}}}{k T}\right)-1
$$

where the short-circuit current $J_{\text {sc }}$ is determined by the incident photon flux and typically equals around $40 \mathrm{~mA} \mathrm{~cm}{ }^{-2}$ for standard sun-simulator conditions.

It is common to map the potential open-circuit voltage across the junction, e.g. to visualize edge passivation problems. It can be acquired at different stages of fabrication to observe the quality of the junction alone and its degradation during subsequent processing [11]. The resolution determined by the microwave beam of half a millimeter diameter is sufficient on bulk mono- or polycrystalline silicon cells.

On the other hand, carrier dynamics in solar cells are often investigated by the transient photovoltage technique. It is noteworthy that in this case, the apparent decay lifetime depends on the light intensity used for generation or initial carrier density, as has been mentioned by some authors [6]. It was also widely accepted since the pioneer work [4] that rising transients were abrupt compared to falling transients which is untypical for exponential decay. Decay constants derived by this technique were therefore associated 
to a particular illumination intensity [5]. A recent review on the dependence of a first order decay constant on open circuit voltage was published by Kiermasch et al. [12]. In parallel, more sophisticated models of the rise and decay curves, involving different time constants [7], were developed.

\section{Adapting the Kane-Swanson formalism to large signal voltage transients}

\subsection{Carrier decay differential equation}

The carrier decay addressed by the Kane-Swanson formalism is not an exponential decay such as SRH recombination described by a time constant. It is caused by a forward diode current that results from the forward bias of a junction under illumination, and is due to carrier diffusion in the neutral zones of the junction. The key parameter of this forward current is the emitter saturation current $J_{\mathrm{oe}}$ which is determined by the diffusion length and the width of the junction. The KS equation suggests that for small signal variation, $d n \ll n$, the decay can be treated as first order exponential decay, with a decay time constant that is inversely dependent on the carrier density. It is clear that if the time constant is not constant but depends itself on the carrier density, the exponential decay is at best a small signal approximation valid for a small range of carrier density variation. We propose to derive a decay law of non-exponential decay with general validity even for large carrier density fluctuation. As in the demonstration of the Kane-Swanson equation, Auger, SRH, and radiative recombination are grouped together in $\tau_{b}$ of eq. 1 and are neglected for the base region. Here the KS formalism is briefly resumed and will be applied to the modeling of/TPV curves.

The band diagram of a typical photovoltaic junction, under illumination and KFM surface potential measurement, is shown on fig. 1.

It is a typical asymmetric junction with a large low doped absorber called "base" and a thin highly doped counterpart provided with an ohmic contact, referred to as "emitter". It is shown without front contact, either because it is an incomplete partial junction or the Kelvin probe is away from one of those electrodes called "finger". We shall consider that the photogenerated minortiy carriers are all in the neutral region of the base and the fraction of carriers about to recombine in the emitter is negligible. Furthermore, the recombination current of the emitter shall be the only source of carrier loss whereas in the neutral region of the base, the bulk recombination effects SRH, Auger and radiative recombination are neglected. Consequently, in the base, the pseudo-Fermi level and minority carrier dénsity are constant.

If the junction was provided with a front contact, the approximation of constant minority carrier density in the base would still hold: due to asymmetry, low bulk recombination and high mobility in the base, the pseudo-Fermi level would decay only in the very proximity of the front electrode. Therefore and because of the small fraction of surface covered by front contacts, the carrier density in the base could still be considered constant and the respective recombination current would be much smaller than the one 


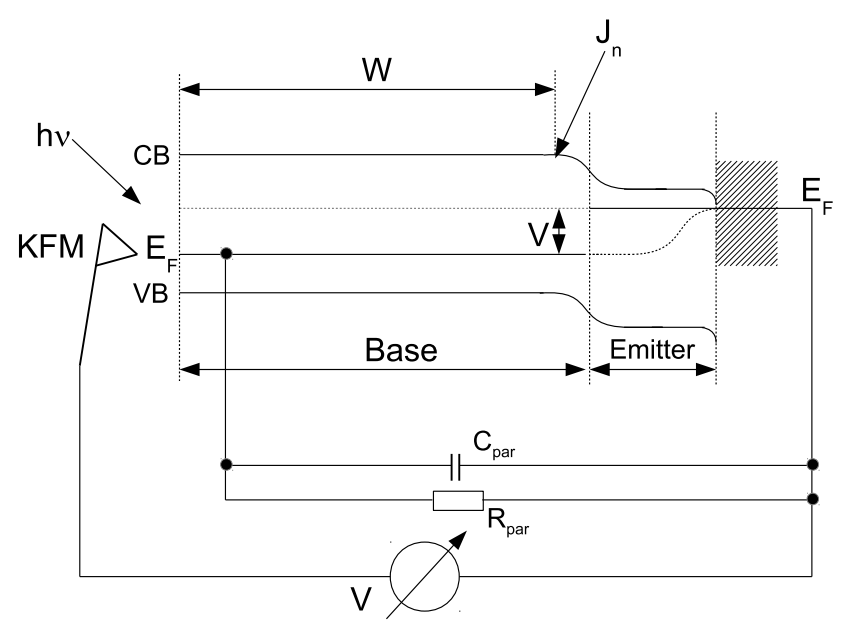

Figure 1. Band structure of a photovoltaic junction under illumination: it is assumed that the minority carrier density is constant throughout the base because of its relatively low doping, negligible bulk recombination and high mobility. The main carrier loss mechanism is the recombination current $J_{n}$ at the boundary between the neutral region of the base and the space charge zone at depth $W$.

of the back emitter. For simplicity, we also renounce to indicating front surface fields in the band structure.

Note that two parasitic parallel components, a capacitor and a resistor, are included. The capacitance to be added is basically the geometric constant capacity, whereas the resistor describes leakage paths. A more detailed description of charge leakage in particular for Perovskite has been presented by Tvingstedt et al. [13].

The continuity equation is written for the base region, neglecting bulk recombination mechanisms:

$$
\frac{d \Delta n(x, t)}{d t}=G(x, t)-D_{n} \frac{d^{2} \Delta n(x, t)}{d x^{2}}
$$

Since the bulk recombination in the neutral base region is neglected, the minority carrier density is constant $\Delta n(x)=\Delta n_{\text {avg }}$ in the neutral area from 0 to $W$. Integrating over the junction depth $\mathrm{W}$ yields

$$
W \frac{d \Delta n_{\mathrm{avg}}(t)}{d t}=\int_{W} G(x, t) d x-\left.D_{n} \frac{d \Delta n(x, t)}{d x}\right|_{x=W}
$$

The last component is the diffusion current and is set equal to the electron recombination current of the emitter at the boundary of the neutral base and the space charge zone:

$$
D_{n} \frac{d \Delta n(W, t)}{d x}=\frac{J_{\mathrm{oe}}}{q} \frac{n(W, t) p(W, t)-n_{\mathrm{i}, \mathrm{eff}}^{2}(W, t)}{n_{\mathrm{i}, \mathrm{eff}}^{2}(W, t)}=\frac{J_{\mathrm{oe}}}{q}\left[\exp \left(\frac{e V}{k T}\right)-1\right](5)
$$

With the same approximations also made in [10] yielding the Kane-Swanson equation, i.e. 


$$
\begin{aligned}
& n \approx \Delta n \\
& p \approx N_{\mathrm{dop}}+\Delta n \gg n_{\mathrm{i}, \mathrm{eff}}^{2}
\end{aligned}
$$

Renaming the integrated generation $g$ and assuming that the density labeled "average" is roughly constant over the junction depth $W$ leads to

$$
\frac{d \Delta n}{d t}=g-\frac{J_{\mathrm{oe}}}{q W} \frac{\Delta n\left(N_{\mathrm{dop}}+\Delta n\right)}{n_{i}^{2}}=\frac{J_{\mathrm{oe}}}{q W}\left[\exp \left(\frac{e V}{k T}\right)-1\right]
$$

Showing clearly that there is a component of overexponential decay, since for $\Delta n \gg N_{\text {dop }}$, it simplifies to

$$
\frac{d}{d t}\left(\frac{\Delta n}{n_{i}}\right)=\frac{g}{n_{i}}-\frac{J_{\mathrm{oe}}}{q W n_{i}}\left(\frac{\Delta n}{n_{i}}\right)^{2}
$$

whereas exponential decay is dominant for $\Delta n<N_{\text {dop }}$, simplifying eq. 7 to

$$
\frac{d}{d t}\left(\frac{\Delta n}{n_{i}}\right)=\frac{g}{n_{i}}-\frac{J_{\mathrm{oe}} N_{\mathrm{dop}}}{q W n_{i}^{2}} \frac{\Delta n}{n_{i}}
$$

The small signal approximation of eq. 1 with an effective exponential decay constant inversely depending on the carrier density $\Delta n$, as found in the literature and applicable to QSS conditions, can easily be derived from eq. 7 by writing

$$
\frac{1}{\tau_{\text {eff }}}=\frac{1}{\Delta n} \frac{d \Delta n}{d t}=-J_{\mathrm{oe}} \frac{N_{\mathrm{dop}}+\Delta n}{q W n_{i}^{2}}
$$

if furthermore the generation is set to zero for modeling only the decay branch.

If surface photovoltage (SPV) transients were erroneously interpreted as exponential decay beyond the validity of the small signal approach $d \Delta n \ll \Delta n$, the lifetime would apparently depend on generation intensity or initial carrier density and the fit with a simple exponential decay would be unsatisfying. If a SRH-like lifetime was derived from such curve fitting, different time constants would also be obtained for rise and fall transients which would give rise to somewhat speculative interpretations.

In this work we shall solve the general case for large signal decay curves by computing the carrier decay numerically.

\subsection{Equivalence of optical and electrical injection}

While studying decay curyes of TMO:Si hybrid solar cells with different injection level and under optical and electrical injection (c.f. section 4.1), the following observations have been madè:

1) Optical generation and electrical injection lead to identical voltage transients after switching off the excitation source at $t=0$. Fig. 2 (a) shows the voltage transients on a TMO solar cell obtained by illuminating with different laser intensities and shutting off the laser at $t=0$, whereas fig. 2 (b) shows the voltage transients after applying 

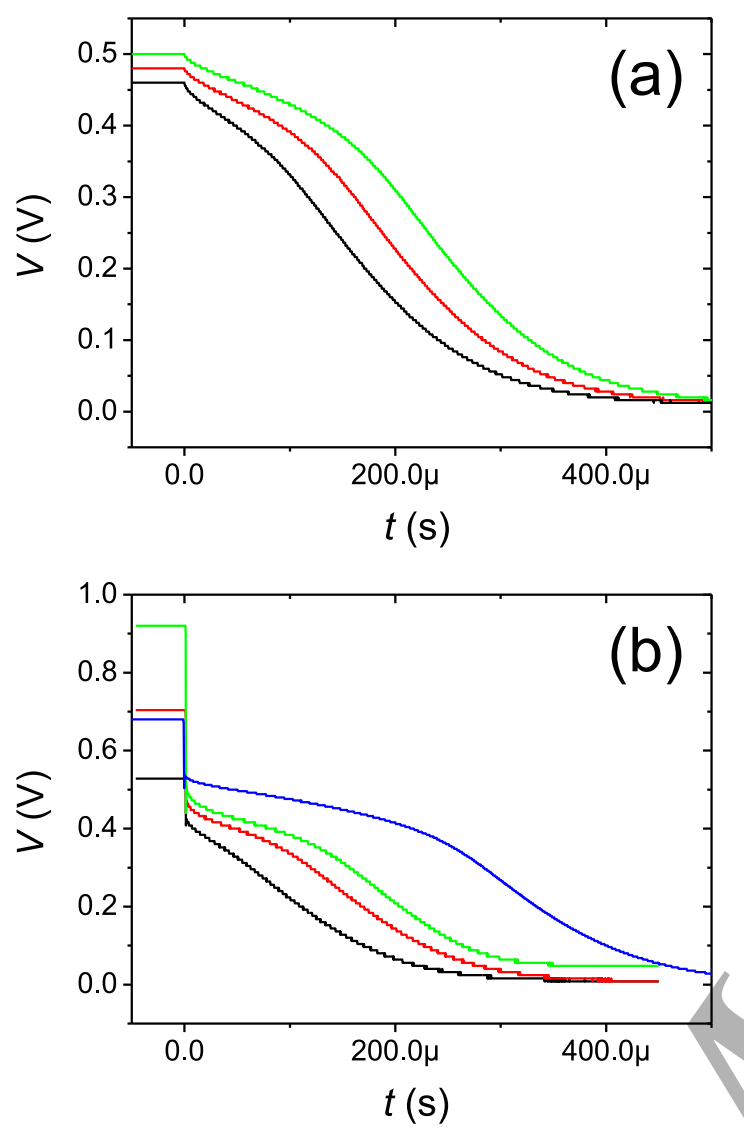

Figure 2. Voltage transient of a TMO solar cell on substrate doped $N_{\text {dop }}=10^{14} \mathrm{~cm}^{-3}$; (a) optical injection switched off at $t=0$, with intensities $5,10,20 \mathrm{~mW}$; (b) electrical injection switched off at $t=0$, with currents of $1.33,3.33,6$ and $30 \mathrm{~mA}$.

forward current and turning it off. The only difference is the additional voltage drop across the contact series resistance for electrical injection.

2) The curves of lower injection are to a large extent superposable to the lower portion of curves with higher injection by shifting their $t=0$ origin so that the starting value of the voltage is identical to the voltage of the curve with higher injection at a time $t>0$. Therefore we will analyze only the curves with the highest injection.

Note that fig. 2 (b) contains one transient with a higher excitation intensity for $30 \mathrm{~mA}$ whose optically excited equivalent could not be reached with our laser intensity. The condition for equal carrier generation $g$ by a current $I$ or by optical generation with power $p$ applied to an area $A$ is :

$$
\begin{aligned}
& g=\frac{I}{q W A}=\frac{p}{h \nu W A} \\
& I[\mathrm{~A}]=p[\mathrm{~W}] \lambda[\mathrm{nm}] / 1240
\end{aligned}
$$


and was roughly satisfied for the applied laser powers and injection currents.

\section{Generally valid solution of the carrier decay differential equation}

We shall solve the differential equation 7 so that it covers all ranges of carrier density from overexponential decay at strong injection $\Delta n>N_{\text {dop }}$, exponential decay for $N_{\text {dop }}>\Delta n$, to the approach of zero voltage.

\subsection{Steady state}

The emitter saturation current $J_{\mathrm{oe}}$ is obtained from the steady state where the carrier density is constant by setting $d n / d t=0$ in eq. 7 :

$$
J_{\text {oe }}=g q W \exp \left(-\frac{e V}{k T}\right)
$$

With optical or electrical excitation $g$ defined by eq. 11 .

\subsection{Infinitesimal decay computation}

A voltage transient can be computed by solving the general differential eq. 7 infinitesimally. The carrier density as function of voltage is

$$
\Delta n=\frac{-N_{\mathrm{dop}}}{2}+\sqrt{\frac{N_{\mathrm{dop}}^{2}}{4}+n_{i}^{2} \exp \left(\frac{e V}{k T}\right)}
$$

Then we compute the infinitesimal change in charge density $d \Delta n$ due to diffusion current:

$$
d \Delta n=d t\left[g-\frac{J_{\mathrm{oe}}}{q W}\left[\exp \left(\frac{e V}{k T}\right)-1\right]\right]
$$

By the derivative of eq. 14, the infinitesimal voltage change

$$
d V=\left(\frac{d \Delta n}{d V}\right)^{-1} d \Delta n
$$

is computed and added to the previous value.

\section{Application to solar cells}

Open circuit photovoltage decay curves are measured on the samples shown in fig. 3. 

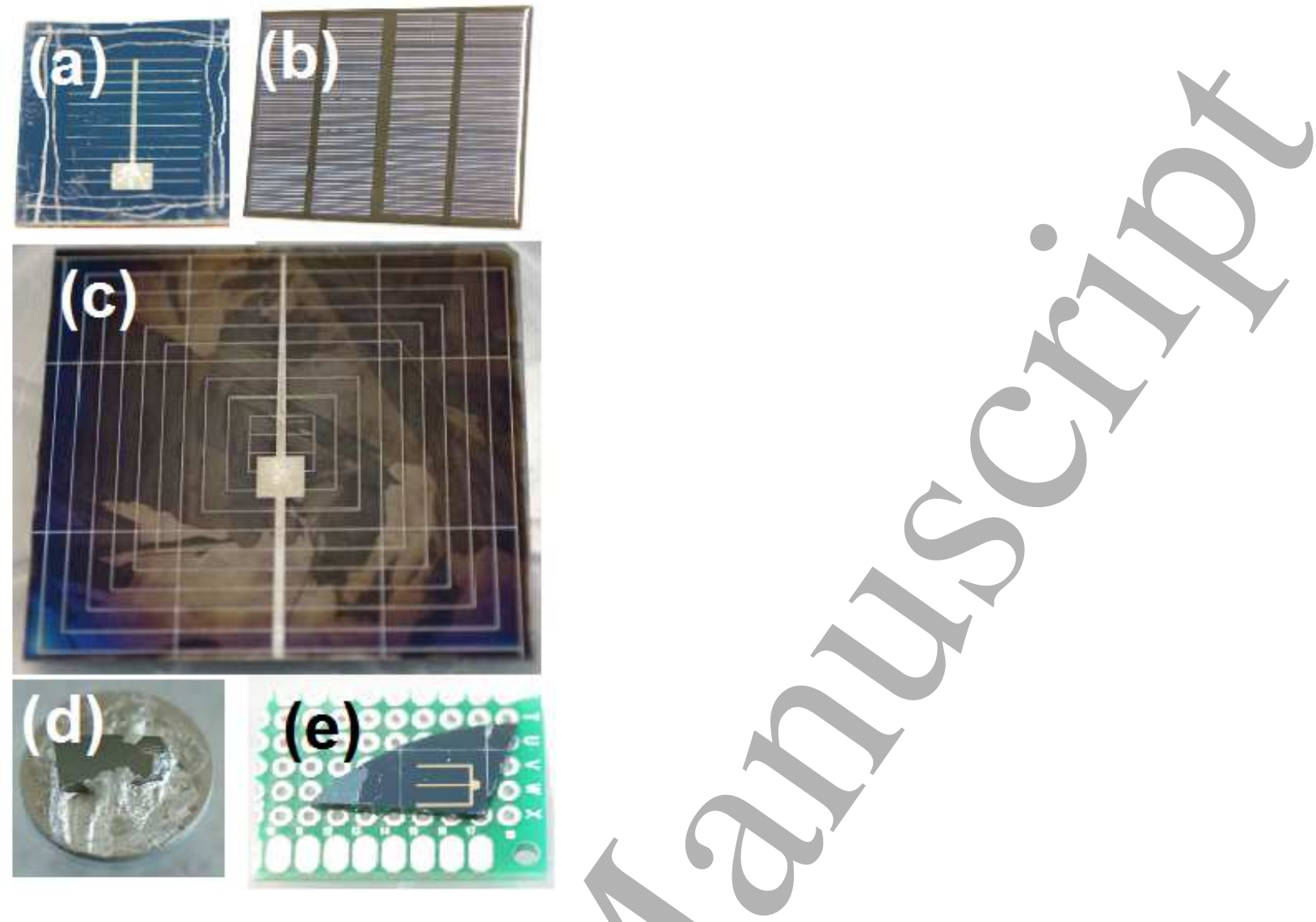

Figure 3. Solar cell samples used in transient voltage spectroscopy: (a) TMO:Si, (b) monochrystalline silicon 12 volt solar panel, (c) monochrystalline silicon cell with surface passivation and antireflection coating, 11 percent efficiency, (d) methylammonium lead iodide Perovskite (MAPbI) junction, (e) CIGS cell.

\subsection{Transition metal oxide / siticon hybrid cell}

A hybrid silicon:transition metal oxide (Si:TMO) solar cell was fabricated by deposing $\mathrm{MoO}_{3}$ onto n-type substrate doped $N_{\text {dop }}=10^{14} \mathrm{~cm}^{-3}$, shown on fig. 3 (a)). Voltage transients have been acquired macroscopically by oscilloscope under electrical injection.

The decay curve for injection with $I / A=50 \mathrm{~mA} \mathrm{~cm}^{-2}$ along with its modeling is shown in fig. 4. The initial voltage after turning off the current is $V=0.508 \mathrm{~V}$, yielding from eqs. 11 and 13 a saturation current $J_{\mathrm{oe}}=1.5 \cdot 10^{-10} \mathrm{~A} \mathrm{~cm}^{-2}$. The red dashed curve is the voltage transient modeled by taking into account the diffusion current and the diffusion capacitance. It shows a steep onset for overexponential decay and passes into a linear slope for the exponential decay regime (since the voltage is roughly the logarithm of the carrier density). Surprisingly, the experimental curve deviates from the diffusion current model below a certain voltage where its slope becomes steeper again. A way to account for this increase of the slope at lower voltage is taking into account a parallel parasitic resistor R. A relatively low parallel resistor of $6.7 \mathrm{k} \Omega \mathrm{cm}^{2}$ had indeed been measured at reverse bias. This resistance can make the slope steeper at lower junction voltage because the carrier density decreases exponentially with voltage but the current 


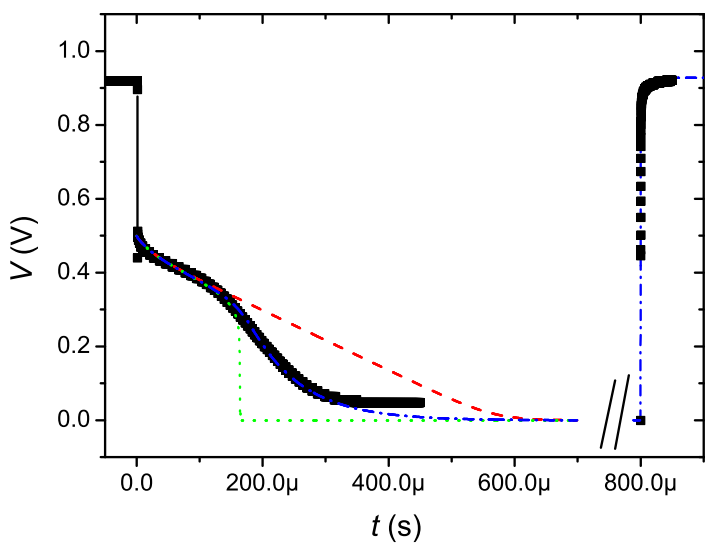

Figure 4. Transient of TMO:Si solar cell doped $N_{\text {dop }}=10^{14} \mathrm{~cm}^{-3}$ and electrically injected: black squares experimental, red dashes : infinitesimally computed decay curve with diffusion current and diffusion capacifance, green dots: computed decay curve also including a parallel resistor $R=6.7 \mathrm{k} \Omega$, and blue dash-dot: computed curve also including both the parallel resistor $\mathrm{R}$ and a parallel capacitance $C=12 \mathrm{nF}$. Above $t=0.8 \mathrm{~ms}$, the rising transition is shown for both experimental data and modeled with the same parameters as the decay, including furthermore the generation term.

through the resistor only decreases linearly. Less there are minority carriers in the base, steeper the decay becomes, resulting in a charge breakdown effect.

Mathematically the parallel resistance $R$ contributes to the charge decay, eq. 15 , as

$$
d \Delta n=d t\left[g-\frac{J_{\mathrm{oe}}}{w q}\left[\exp \left(\frac{e V}{k T}\right)-1\right]-\frac{V}{R A w q}\right]
$$

The subsequent treatment is the same, $d V$ is obtained by eq. 16 . The effect of the parallel resistance is shown on fig. 4 as the green curve for $\mathrm{R}=6.7 \mathrm{k} \Omega$. The reason why experimentally the breakdown effect is less pronounced in the experimental curve may be explained by the presence of a parallel parasitic capacitance $C$ that smoothes out the queue of the decay and that we will also add to the model by adapting eq. 14:

$$
\Delta n=\frac{V C}{A w q}-\frac{N_{\mathrm{dop}}}{2}+\sqrt{\frac{N_{\mathrm{dop}}^{2}}{4}+n_{i}^{2} \exp \left(\frac{e V}{k T}\right)}
$$

The charge stored in the capacitor has also been converted into a density by dividing by the junction volume and elementary charge. In the computation of $\mathrm{dV}$, the derivative $d n / d V$ changes accordingly and so does eq. 16.

With the resistor and capacitor in parallel, we can model the experimental transient quite well, illustrated in fig. 4 as the blue dash-dotted curve for a $C=12 \mathrm{nF}$ capacitor and $R=6.7 \mathrm{k} \Omega$. A similar capacitance value of $C=7 \mathrm{nF}$ had been found by $\mathrm{AC}$ impedance measurement. A deviation between model and measurement occurs only at the lowest part of the queue. Possibly the model based on fixed parallel resistors 
and capacitances is not enough sophisticated and in reality the differential capacitance needed for a more accurate model of the parasitic elements would be by itself voltage dependent.

Finally, with the determined parameters it is also possible to model the rising edge of the voltage transient. The start value is set to zero and the generation according to eq. 11 is applied for $t>0.8 \mathrm{~ms}$, shown in fig. 4 . The successful modeling of the rising edge clearly demonstrates that it is an inherent feature of the overexponential decay law to yield asymmetry between falling and rising transients despite the same time constant, in contrast to exponential decay law.

We emphasize that the parameters can conveniently be found one after the other:

- In the beginning of the decay where the overexponential and exponential decay approximations are valid, $J_{\mathrm{oe}}$ and $N_{\text {dop }}$ can be determined.

- The value of the resistor determines the onset of the breakdown effect and can also be measured at small bias.

- The value of the parallel capacitor is progressively increased in curve fitting to soften the breakdown effect and adapt the lower part of the decay to the experimental data. It can be measured at a small AC bias.

\subsection{Commercial solar cell}

We continue the study on a randomly purchased commercial solar cell shown on fig. 3 (b).

The panel is composed of 48 small cells of a surface $A=1.32 \mathrm{~cm}^{2}$ each, which are apparently connected in series to reach the output voltage rated $12 \mathrm{~V}$. This has been revealed by a dark $\mathrm{I}(\mathrm{V})$ curve under forward bias that has a unity ideality factor when dividing the panel voltage by 48 . The same $\mathrm{I}(\mathrm{V})$ measurement also yields an emitter saturation current density of $J_{\mathrm{oe}}=2.60 \cdot 10^{-7} \mathrm{~A} \mathrm{~cm}^{-2}$ given by eq. 13. Furthermore, measurements on the panel revealed a capacitance of $9.7 \mathrm{nF}$ for the panel, corresponding to a capacity per surface $C=353 \mathrm{nF} \mathrm{cm} \mathrm{cm}^{-2}$, and a parallel resistance of $200 \mathrm{k} \Omega$ for the panel corresponding to a surface resistivity of $R=5.5 \mathrm{k} \Omega \mathrm{cm}^{2}$.

Fig. 5 shows the voltage transients for $0.762 \mathrm{~mA}$ injection current leading to a starting voltage of $9.6 \mathrm{~V}$ for the panel, corresponding to $0.2 \mathrm{~V}$ per cell. The red curve is the infinitesimal computation taking into account the differential diffusion conductance and capacitance. Due to the relatively high emitter saturation current, the ratio between differential conductance and differential capacitance is such that the decay is abrupt. Varying the width of the junction from $W=100 \mu \mathrm{m}$ to $W=300 \mu \mathrm{m}$ has no noticeable effect. Likewise, the dopant density can be varied from $N_{\text {dop }}=10^{14} \mathrm{~cm}^{-3}$ to $N_{\text {dop }}=10^{17} \mathrm{~cm}^{-3}$ without noticeable effect on the modeled decay curve.

Only when the parallel capacitance of $C=353 \mathrm{nF} \mathrm{cm} \mathrm{cm}^{-2}$ is included, a relatively close curve fit is obtained (green dotted line). If furthermore the parasitic resistance is included into the model, the fit becomes perfect. However, the value had to be slightly 

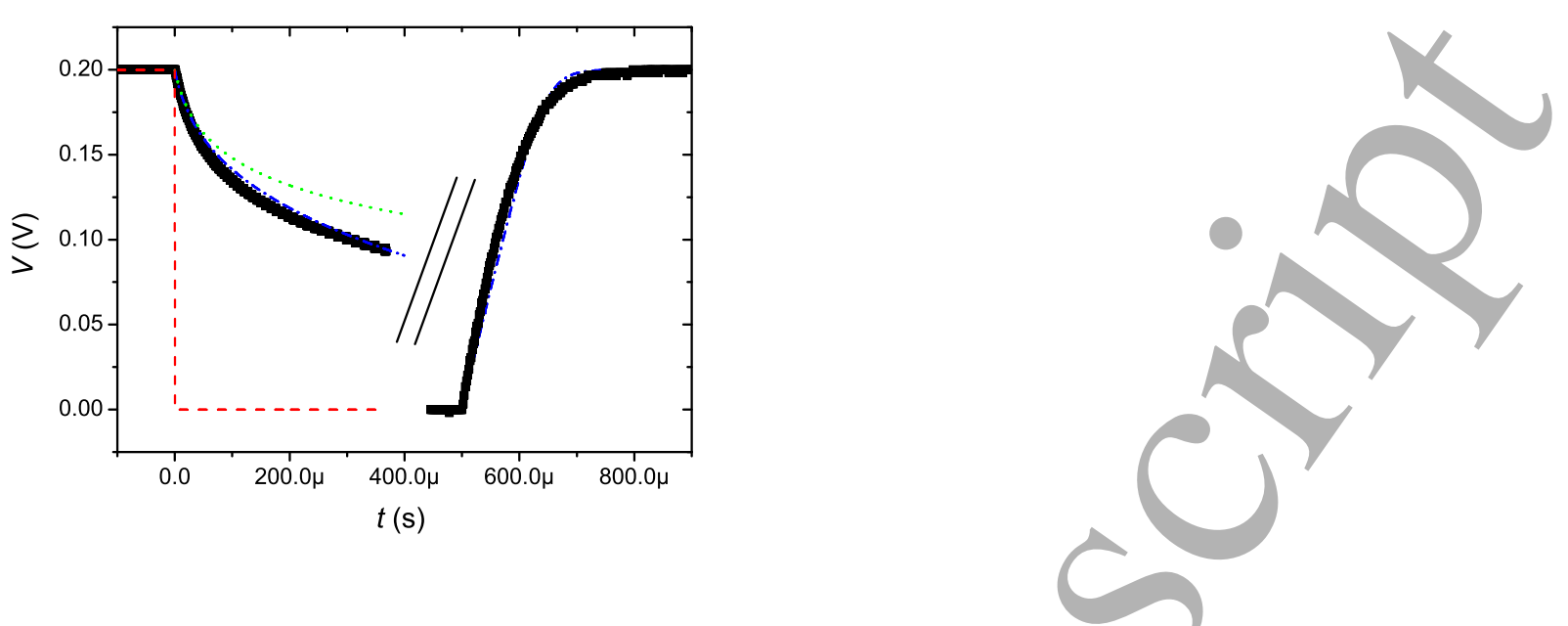

Figure 5. Voltage transient of a commercial solar cell, measured by injecting a forward current of $0.76 \mathrm{~mA}$ yielding $0.2 \mathrm{~V}$ per cell, and turning off the injection at $t=0$. The voltage is given per cell. Red dashed curve: decay modeled with diffusion capacitance and diffusion conductance, with $J_{\mathrm{oe}}=2.60 \cdot 10^{-7} \mathrm{~A} \mathrm{~cm}-2, W=100 \mu \mathrm{m}$ to $300 \mu \mathrm{m}$ and $N_{\text {dop }}=10^{14} \mathrm{~cm}^{-3}$ to $N_{\text {dop }}=10^{17} \mathrm{~cm}^{-3}$; green dots: modeling that also includes a parallel capacitance $C=353 \mathrm{nF} \mathrm{cm} \mathrm{cm}^{-2}$; blued dash-dotted line: model that also includes $\mathrm{C}$ and $R=3.3 \mathrm{k} \Omega \mathrm{cm}^{-2}$. For $t>0.5 \mathrm{~ms}$ the rise is shown, measured and modeled (blue dash-dotted) with the same parameters as the decay and a generation term according to eq. 11.

lowered to $R=3.3 \mathrm{k} \Omega \mathrm{cm}^{-2}$ versus the $5.5 \mathrm{k} \Omega \mathrm{cm}^{2}$ extracted from measurements performed on the panel.

The decay curve is dominated by diffusion current and the charge carriers stored in the parallel capacitance. The parasitic capacitance of $C=353 \mathrm{nF} \mathrm{cm}-2$ appears large. It is more likely that the manufacturer has deliberately connected a capacitor of some $\mathrm{nF}$ in parallel to the entire panel to prevent the solar cells from radiating switching noise caused by subsequent converters.

Above $t=0.5 \mathrm{~ms}$, the rising transient is modeled with the same parameters. The generation term for electrical injection has been computed from the steady state according to eq. 11. The large parallel capacitance effectively prevents drawing more information about the junction, as has been done for the TMO solar cell where it was possible to confirm the product of doping level and thickness.

\subsection{Silicon cell passivated with nitride and featuring an antireflection layer}

An $\mathrm{Al}_{2} \mathrm{O}_{3}$ passivated silicon cell with 11 percent photoconversion efficiency, as described in [14] and shown on fig. 3(c), was then studied. The photovoltage transients were macroscopically measured by oscillography, with an injection current of $100 \mathrm{~mA}$ corresponding to $4 \mathrm{~mA} \mathrm{~cm}^{-2}$, shown on fig. 6 .

The emitter saturation current needed for the decay modeling was obtained from an $\mathrm{I}(\mathrm{V})$ measurement yielding $J_{\mathrm{oe}}=2.137 \cdot 10^{-7} \mathrm{~A} \mathrm{~cm}^{-2}$ and an ideality factor $n=1.992$. 

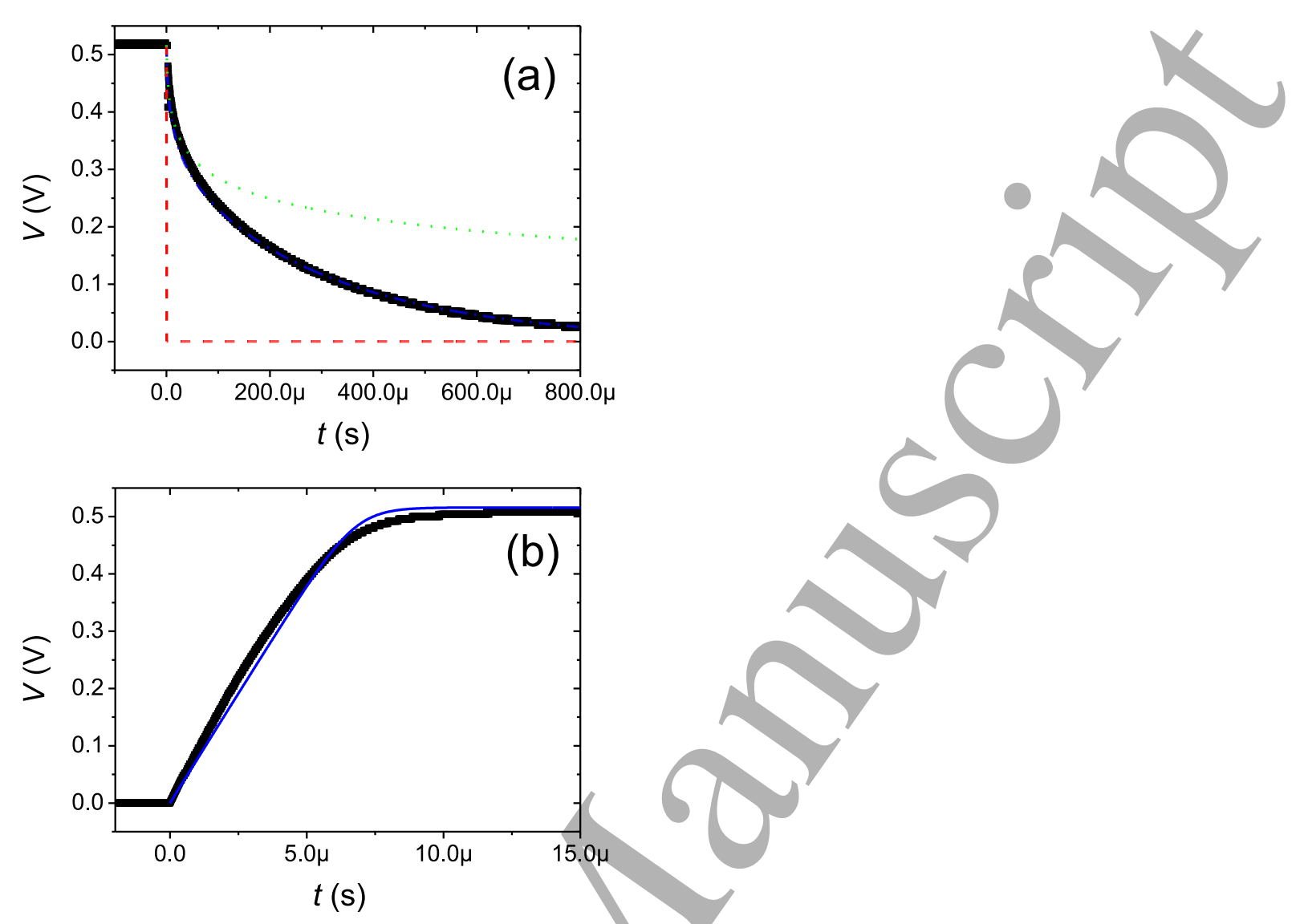

Figure 6. Voltage transient of a monocrystalline silicon solar cell of fig. 3 (c); (a) decay curve, black squares: measured, red dashes: differential conductance and diffusion capacitance only, green dots: including a parasitic capacitance of $90 \mathrm{nF} \mathrm{cm}^{-2}$, blue dash-dot : including parasitic capacitance and resistance of $3.3 \mathrm{k} \Omega \mathrm{cm}^{2}$; (b) rise curve, modeled with the same parameters as the decay curve including $\mathrm{R}$ and $\mathrm{C}$.

The parasitic values obtained by small signal measurements at $100 \mathrm{mV}$ amplitude and freqencies from 1 to $10 \mathrm{kHz}$ were $C=40 \mathrm{nF} \mathrm{cm} \mathrm{cm}^{-2}$ and $R=5 \mathrm{k} \Omega \mathrm{cm}^{2}$.

The decay of fig. 6 (a) was first modeled with the differential conductance and differential capacitance only (red dashes) for a base thickness of $W=360 \mu \mathrm{m}$ and base doping levels ranging from $5 \cdot 10^{14}$ to $1 \cdot 10^{17} \mathrm{~cm}^{-3}$. However, for the given saturation current, the diffusion capacitance is so small that the decay appears abrupt irrespective of the doping in the above range of doping levels. Next, the effect of the parasitic capacitance was taken in account. It was necessary to increase this capacitance to $90 \mathrm{nF} \mathrm{cm}^{-2}$ to obtain the decay of the green dotted line, without crossing the experimental decay curve. In the last step, a parallel shunt resistance was added, yielding the blue dash-dotted line that perfectly fits the experimental data. The resistance value had to be lowered to $3.3 \mathrm{k} \Omega \mathrm{cm}^{2}$ vs. the previously measured $5 \mathrm{k} \Omega \mathrm{cm}^{2}$ to obtain a perfect fit. 
The same parameters were then used to model the rising voltage transient, shown on fig. 6 (b) with a significantly smaller timescale, by setting the initial voltage to zero and including a generation term according to eq. 11. A fairly good fit was immediately obtained.

As in the previous silicon solar cell, the differential diffusion capacitance is masked by the parasitic values. Therefore, neither base thickness nor doping level can be determined from the modeling, but the transients with their asymmetry between falling and rising edge can be modeled accurately.

Since the I(V) curve has an ideality factor of 2 , the question arises whether in the previous commerical solar cell, there might be 2 parallel sets of 24 cells in series rather than 48 cells in series, if the ideality factor was also 2 instead of unity.

\subsection{Perovskite photojunction studied by KFM}

As third sample, an inverted 3D Perovskite junction, consisting of only the layers ITO, methylammonium lead iodide (MAPbI3) and PCBM, without top contact, has been studied. The surface photovoltage transient of such an incomplete solar cell can be studied only by the Kelvin probe and under optical excitation. Coincidentally, the surface photovoltage decay times were found to be compatible with the response of our Kelvin microscopy (KFM) setup with its $300 \mathrm{~Hz}$ bandwidth. A piece of the junction, glued by conductive epoxy on a steel plate, is shown on fig. 3 (d).

A surface photovoltage curve was acquired by KFM on a Perovskite photovoltaic junction under laser illumination of $640 \mathrm{~nm}$ wavelength and switched at $2.5 \mathrm{~Hz}$, with $1 \mathrm{~mW}$ output power, and directing the beam without further optics onto an area $A=0.05 \mathrm{~cm}^{2}$ surrounding the KFM probe. The resulting SPV transient curve is shown on fig. 7 .

The aim of the following characterization shall be the attribution of parameters in the simple picture of a pn-junction such as the intrinsic carrier density, the doping level, or the junction width that in a monocrystalline silicon solar cell would roughly equal the substrate thickness.

We obtain a steady state forward voltage of $V=0.46 \mathrm{~V}$ yielding $J_{\text {oe }}=2$. $10^{-10} \mathrm{~A} \mathrm{~cm}^{-2}$ from eq. 13. The voltage decays more than linearly, showing that the carrier decay is overexponential. This can be due to strong carrier injection $\Delta n \gg N_{\text {dop }}$ or possibly result from interaction of a constant capacity with the differential conductance of the diffusion current. In the former case, the approximation of eq. 8 is applicable, and the decay modeling is performed by setting the (negligible) dopant density $N_{\text {dop }}$ to zero, allowing to determine

$$
\frac{J_{\mathrm{oe}}}{q W n_{i}}
$$

Therefore, for a given $J_{\text {oe }}$ we can further obtain the product of the intrinsic carrier density and the junction width but neither of the factors individually. Under this 

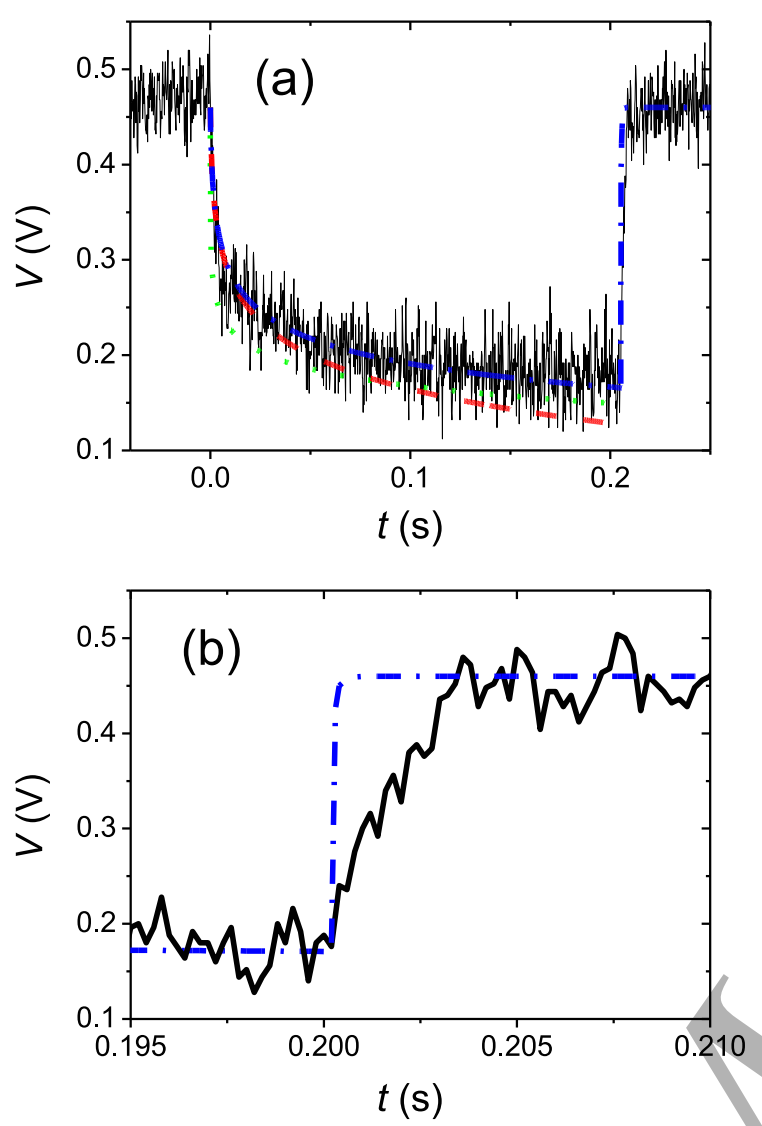

Figure 7. Surface photovoltage transients (a) measured by KFM on Perovskite sample (black) for a laser modulated with $2.5 \mathrm{~Hz}$. Red dashed curve: modeling of overexponential decay function dominated by diffusion capacitance $W=0.01 \mathrm{~cm}$ and $n_{i}=3 \cdot 10^{11} \mathrm{~cm}^{-3}$, green dotted: decay model for negligible diffusion capacitance and parallel capacitance $C=500 \mathrm{hF} \mathrm{cm}^{-2}$, and blue dash-dotted: model including both diffusion capacitance and parallel capacitance; (b) zoom of the rising photovoltage transient (black solid) and its modeling (blue dash-dot) using the same parameters as for the decay.

hypothesis, a particular solution is found with a thickness $W=100 \mu \mathrm{m}$ and intrinsic carrier density $n_{i}=3 \cdot 10^{11} \mathrm{~cm}^{-3}$, shown by the red dashed line of fig. 7 .

For the latter case, the interaction of the parallel capacitance with the diffusion conductance, the decay is modeled with $C=500 \mathrm{nF} \mathrm{cm}^{-2}$, the previously determined $J_{\text {oe }}$, and with negligible diffusion capacitance, shown by the green dotted curve of fig. 7 (a). Finally, the blue dash-dotted curve contains both contributions and yields a satisfying fit, whereas neither individual contribution fits all over the decay no matter how the values are chosen. This confirms the hypotheses that the strong carrier injection regime is reached here, but also shows a large parasitic capacitance which might be due to the 3D character of the Perovskite junction. However, the accuracy of the parameters cannot be given with the approximate manual decomposition into two components 
(diffusion and external capacitor). The quality of the curve fit also depends very sensibly on the description of the $I(V)$ curve by the exponential function. On this incomplete solar cell, an $I(V)$ curve was not recorded and the saturation current was only determined from the open circuit voltage under one illumination intesity, assuming a unity ideality factor of the diode characteristics. Any deviation from this idealized behavior would affect the modeling of the decay and the parameters, which might explain the unusually high parasitic capacitance. Theroretically the ideality factor can be obtained from recording open circuit voltage versus illumination intensity, but this requires the laser power to be reliably controlled over a certain range of intensities.

The transient is also computed for the rising edge with the same values as for the decay, an optical generation term according to eq. 11, and a non-zero starting value for the SPV since it was not allowed to decay to a negligible value. The modeling reproduces the asymmetry between falling and rising edge fairly well. The model actually predicts a faster rise than the experimental transient as shown on the fast timescale of fig. 7 (b). The $3 \mathrm{~ms}$ rise time difference is exactly due to the $300 \mathrm{~Hz}$ bandwidth limit of the KFM.

\subsection{CIGS solar cell}

The last sample is a CIGS solar cell, composed of a junction of 2 microns of CIGS with $50 \mathrm{~nm}$ of $\mathrm{CdS}$, shown in fig. 3 (e). Its fabrication is described by Ayachi et al. $[15,16,17,18]$. It is studied by oscilloscopy under electrical injection of $2.5 \mathrm{~mA}$ for a sample surface of $0.45 \mathrm{~cm}^{2}$.

A $I(V)$ curve reveals an emitter saturation current $J_{\text {oe }}=2.9 \cdot 10^{-6} \mathrm{~A} \mathrm{~cm}^{-2}$ and an ideality factor of $n=2.37$. For these diode characteristics, the parasitic parallel values are difficult to measure even under $\mathrm{AC}$ small signal and were estimated to $C=30 \mathrm{nF} \mathrm{cm} \mathrm{cm}^{-2}$ and $R=5 \mathrm{k} \Omega \mathrm{cm}^{2}$.

Falling and rising voltage transients together with modeled curves are shown on fig. 8 (a) and (b).

The decay is overexponential as for the previous sample. The black dashed curve shows the decay with the diffusive capacitance and diffusive conductance only, yielding the intrinsic carrier density $n_{i}=1.2 \cdot 10^{13} \mathrm{~cm}^{-3}$. The blue dash-dotted curve contains furthermore the parasitic capacitance. Its value had to be adjusted to $C=40 \mathrm{nF} \mathrm{cm}-2$ during the curve fitting and it reveals that the parasitic resistance is not needed in the model, it can be set to infinity. The rise is obtained with the same parameters as the decay and by including a generation term according to eq. 11. Due to the known thickness, the intrinsic carrier density can be obtained directly in contrast to the previous sample where only the product of thickness and intrinsic density is determined. Again, the large ideality factor makes it difficult to disentangle the parasitic values at low bias from the $\mathrm{I}(\mathrm{V})$ caracteristics. 

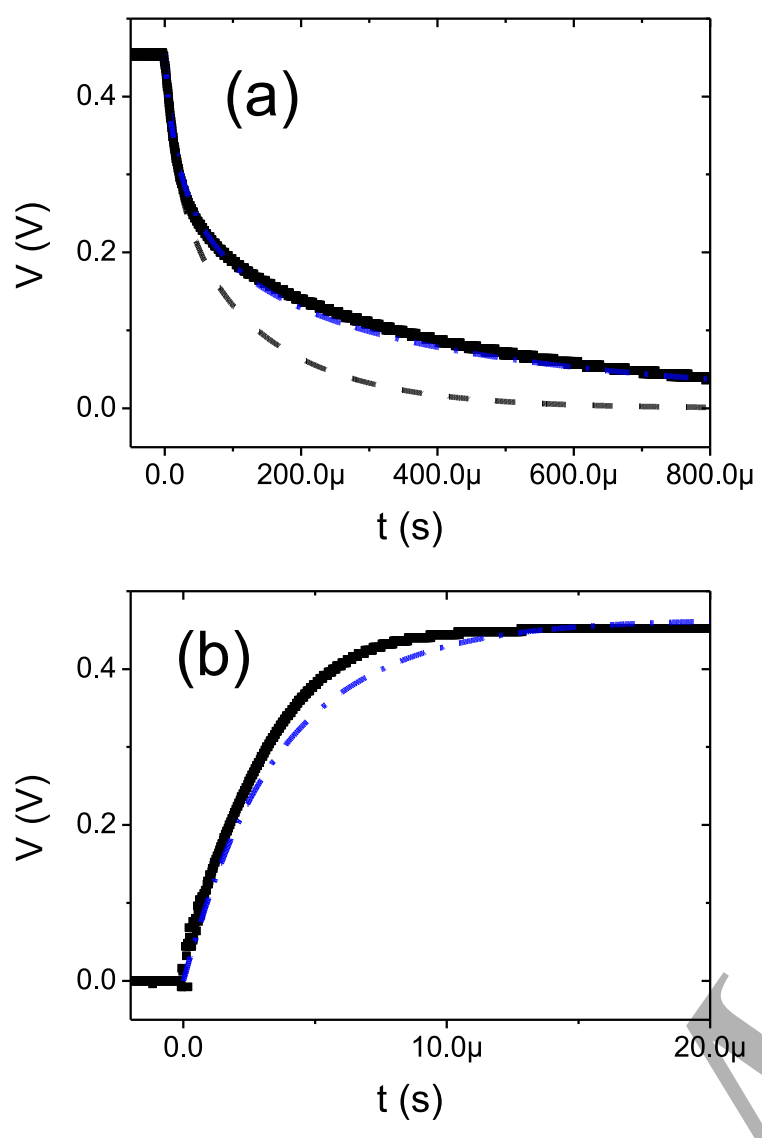

Figure 8. Voltage transients: (a) decay curve of the CIGS sample of fig. 3 (e). The curve fit uses the values $J_{\mathrm{oe}}$ and the ideality factor from the $I(V)$ curve; black dashed: diffusive capacitance and diffusive conductance only, yielding $n_{i}=1.2 \cdot 10^{13} \mathrm{~cm}^{-3}$, $N_{d}=0$; blue dash-dotted curve including furthermore $C=40 \mathrm{nF} \mathrm{cm}^{-2}$; (b) rising transient modeled with the same parameters.

\section{Discussion}

The modeling of SPV decay curves is a powerful method for determining unknown solar junction characteristics. The shape of the decay curve is determined by a differential capacitance and a differential conductance. Candidates of capacitances are the diffusion capacitance which is subdivided into strong and weak injection, and a constant parasitic or geometric parallel capacitance. The two possible conductance mechanisms are the diffusion conductance and the parasitic parallel resistance. Hence, six possible RC combinations may be dominating the shape of the decay over its totality or portions of it. Depending on whether an experimental decay curve extends over one or several regimes dominated by a particular set of differential $\mathrm{R}$ and $\mathrm{C}$ components, the curve fitting allows to eliminate one or more unknown parameters accordingly. Experimentally, we have determined the emitter saturation current $J_{\text {oe }}$ and (where applicable) the ideality factor 
from the steady state SPV under continuous excitation, and estimated the parasitic parallel resistance and capacitance by small signal measurements. These values were used as starting values for the subsequent computation of the decay curve. In the TMO cell, the decay onset is governed by the diffusion capacitance in strong injection regime and diffusion conductance, observed by the overexponential decay. It is followed by the interaction of weak injection diffusion capacitance with the diffusion conductance. The next portion is a breakdown regime, resulting from intercation between weak injection diffusion capacitance and parasitic resistance. This allowed to verify both the base width and the doping (which in this case were known a priori because the cell was fabricated in-house by the co-authors). The two silicon cells are dominated by the parasitic capacitance interacting with the diffusion current. Therefore neither the doping nor the base width could be determined. Nevertheless the modeling correctly reproduced the transients based on differential conductance and parallel parasitic components. In the Perovskite and CIGS solar cells, the prevailing mechanisms are the diffusion capacitance in the strong injection range and the diffusion conductance. However, the parasitic constant capacitance of the Perovskite sample is large and it is difficult to disentangle it from the differential diffusion capacitance. The product $W n_{i}$, both parameters of which were unknown for this material, could furthermore be determined. In the CIGS cell, an intrinsic carrier density $n_{i}$ could be assigned since its thickness $W$ is known.

\section{Experimental}

Surface voltage transients on the complete Si:TMO, Si and CIGS solar cells have been measured directly by a digital storage oscilloscope connected to their contacts, whereas the Kelvin probe was used on the partial Perovskite junction. The optical excitation was achieved by a $640 \mathrm{~nm}$ Coherent Obis diode laser modulated by a HP8112A pulse generator. The electrical current,injection was applied by switching a BUZ11A MOSFET by a standard function generator. SPV transients on the Perovskite partial junction have been acquired by AM-KFM based on a Omicron VT-AFM controlled by a Specs AFM controller. The bandwidth of the AM-KFM has been set to $>300 \mathrm{~Hz}$ [19]. The direct measurement of transients is necessarily bandwidth-limited and a rise or fall time constant of less than $3 \mathrm{~ms}$ cannot be interpreted as intrinsic feature of the junction. The oscilloscope was set to average over 256 curves during the acquisition of all transients to improve the signal to noise ratio.

\section{Conclusion and perspectives}

Inspired by photoconductance measurements of the minority carrier decay in solar cells, the underlaying mechanism of the carrier dynamics, a combination of differential capacitance and conductivity, has been identified. Then, the differential equation has been rewritten so that the carrier decay can be computed infinitesimally in the large signal domain rather than in QSS conditions. In addition, the density of 
minority carriers stored in the diffusion capacitance has been converted into a surface photovoltage which can be conveniently measured by the Kelvin probe.

It has been shown on different solar cells that surface voltage transients can be fairly well modeled over a voltage range corresponding to a carrier density variation of 5 orders of magnitude. The asymmetry between falling and rising transition is perfectly accounted for by the modeling.

Depending on whether the carrier dynamics are dominated by the differential capacitance and conductance or by external parasitic values, it is possible to determine different missing key parameters of a photovoltaic junction such as the doping level or the width of the base.

In the present work, the transients were computed infinitesimally, but the junction characteristics have been assumed as idealized (abrubt doping profiles and depleted zones). The only non-ideal behavior taken into account was the determining of a diode ideality factor for the silicon and CIGS solar cells of fig. 3 (c) and (e). All approximations from the very beginning of the Kane-Swanson method have been adopted. As perspective, the validity range can be discussed as has previously been done for photoconductance measurements [10], and numerical methods can be extended to account for real devices similar as has been done for the junction of a bipolar transistor [20]. Regarding the computational improvement, the curve fitting procedure should ultimately be integrated into an automated least square fit routine rather than searching the best parameters manually by trial and error. This would allow to quantify the errors of the fitting parameters and the junction characteristics.

The largest potential of the technique is its combination with Kelvin microscopy (KFM): the surface photovoltage can be measured by the Kelvin probe on partial solar junctions without top contact. This will speed up conquering future potential photovoltaic materials without building a complete solar cell prior to characterization and allow dissociating the impact of the different contacts on the cell performance. Whereas microwave photoconductance measurements have their resolution limited by the beam diameter, KFM offers better resolution, making it suitable for mapping the junction parameters at the scale of light trapping structuring. In this study the Perovskite sample without top contact was coincidentally, by its slow voltage transient, compatible with the native bandwidth of direct KFM measurement. For acquiring faster SPV transients by KFM on any photovoltaic junction, time averaging methods and ultimately pump-probe techniques will have to be applied.

\section{Acknowledgments}

Support from the french Renatech network is gratefully acknowledged.

\section{References}

[1] A. Pockett, G. E. Eperon, T. Peltola, H. J. Snaith, A. Walker, L. M. Peter, and P. J. Cameron. Characterization of planar lead halide perovskite solar cells by impedance spectroscopy, open- 
circuit photovoltage decay, and intensity-modulated photovoltage/photocurrent spectroscopy. J. Phys. Chem. C, 119(7):3456, 2015.

[2] R. Gottesman, P. Lopez-Varo, L. Gouda, J. A. Jimenez-Tejada, J. G. Hu, S. Tirosh, A. Zaban, and J. Bisquert. Dynamic phenomena at perovskite/electron-selective contact interface as interpreted from photovoltage decays. Chem, 1(5):776, 2017.

[3] A. B. Walker, L. M. Peter, and K. Lobato. Analysis of photovoltage decay transients in dyesensitized solar cells. J. Phys. Chem. B, 110(50):25504, 2006.

[4] M. Takihara, T. Takahashi, and T. Ujihara. Minority carrier lifetime in polycrystalline silicon solar cells studied by photoassisted kelvin probe force microscopy. Appl. Phys. Lett., 93(2):021902, 2008.

[5] L. Borowik, H. Lepage, N. Chevalier, D. Mariolle, and O. Renault. Measuring the lifetime of silicon nanocrystal solar cell photo-carriers by using kelvin probe force microscopy and x-ray photoelectron spectroscopy. Nanotechnology, 25(26):265703, 2014.

[6] GZ. Shao, MS. Glaz, F. Ma, HX. Ju, and DS. Ginger. Intensity-modulated scanning kelvin probe microscopy for probing recombination in organic photovoltaics. ACS Nano, 8(10):10799, 2014.

[7] PAF. Garrillo, L. Borowik, F. Caffy, R. Demadrille, and B. Grevin. Photo-carrier multidynamical imaging at the nanometer scale in organic and inorganic solar cells. ACS APPLIED MATERIALS \& INTERFACES, 8(45):31460, 2016.

[8] R. A. Sinton and A. Cuevas. Contactless determination of current-voltage characteristics and minority-carrier lifetimes in semiconductors from quasi-steadystate. Appl. Phys. Lett., 69, 1996.

[9] DE Kane and RM Swanson. Measurement of the emitter saturation current by a contactless photoconductivity decay method. Proc. 18th IEEE Photovoltaic Specialists Conference, 1985.

[10] H. Maeckel and K. Varner. On the determination of the emitter saturation current density from lifetime measurements of silicon devices. Progress in Photovoltaics, 21(5):850, 2013.

[11] T. Pavelka, A. Pap, and GY. Szilagyi. Carrier lifetime measurements in silicon for photovoltaic applications. ECS Transactions, 11(3):331, 2007.

[12] D. Kiermasch, A. Baumann, M. Fischer, V. Dyakonov, and K. Tvingstedt. Revisiting lifetimes from transient electrical characterization of thin film solar cells; a capacitive concern evaluated for silicon, organic and perovskite devices. Energy \& Environmental Science, 11(3):629, 2018.

[13] K. Tvingstedt, L. Gil-Escrig, C. Momblona, P. Rieder, D. Kiermasch, M. Sessolo, A. Baumann, HJ Bolink, and V. Dyakonov. Removing leakage and surface recombination in planar perovskite solar cells. ACS Energy Letters, 2(2):424, 2017.

[14] M. Pawlik. Etude de la passivation de surface du silicium cristallin type $P$ par depot de couches atomiques d'alumine pour application aux cellules solaires a haut rendement. $\mathrm{PhD}$ thesis, Universite Lille 1, 2015.

[15] B. Ayachi, T. Aviles, and J.-P. Vilcot. Innovative solution to avoid glass substrate bending in a chalcopyrite solar cell fabrication process. Thin Solid Films, 653, 2018.

[16] B. Ayachi, T. Aviles, and J.-P. Vilcot. Rapid thermal annealing effect on the spatial resistivity distribution of azo thin films deposited by pulsed-direct-current sputtering for solar cells applications. Appl. Surf. Sci, 366, 2016.

[17] B. Ayachi, T. Aviles, J.P. Vilcot, C. Sion, and P. Miska. Room temperature pulsed-dc sputtering deposition process for cigs absorber layer: material and device characterizations. submitted to Thin Solid Films, 2018.

[18] B. Ayachi. Towards full sputtering deposition process for CIGS solar cells fabrication: from single thin film deposition up to device characterization. PhD thesis, University Lille 1, 2016.

[19] H. Diesinger, D. Deresmes, J.-P. Nys, and T. Melin. Dynamic behavior of amplitude detection kelvin force microscopy in ultrahigh vacuum. Ultramicroscopy, 110:162, 2010.

[20] CY. Kwok. Space-charge and injection capacitances of p-n-junctions from small-signal numericalsolutions. J. Phys. D, 16(12):L263, 1983.

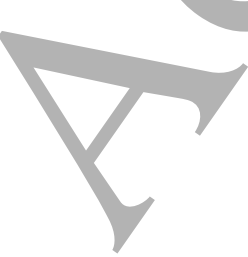

